PROCEEDINGS OF THE

AMERICAN MATHEMATICAL SOCIETY

Volume 136, Number 9, September 2008, Pages 3271-3280

S 0002-9939(08)09407-0

Article electronically published on April 23, 2008

\title{
EQUIFOCALITY OF A SINGULAR RIEMANNIAN FOLIATION
}

\author{
MARCOS M. ALEXANDRINO AND DIRK TÖBEN \\ (Communicated by Jon G. Wolfson)
}

\begin{abstract}
A singular foliation on a complete Riemannian manifold $M$ is said to be Riemannian if each geodesic that is perpendicular to a leaf at one point remains perpendicular to every leaf it meets. We prove that the regular leaves are equifocal, i.e., the end point map of a normal foliated vector field has constant rank. This implies that we can reconstruct the singular foliation by taking all parallel submanifolds of a regular leaf with trivial holonomy. In addition, the end point map of a normal foliated vector field on a leaf with trivial holonomy is a covering map. These results generalize previous results of the authors on singular Riemannian foliations with sections.
\end{abstract}

\section{INTRODUCTION}

In this section, we will recall some definitions and state our main results as Theorem 1.5 and Corollary 1.6 .

We start by recalling the definition of a singular Riemannian foliation (see the book of Molino [9]).

Definition 1.1 (s.r.f.). A partition $\mathcal{F}$ of a complete Riemannian manifold $M$ by connected immersed submanifolds (the leaves) is called a singular Riemannian foliation (s.r.f. for short) if it verifies condition (1) and (2):

(1) $\mathcal{F}$ is a singular foliation; i.e., the module $\mathcal{X}_{F}$ of smooth vector fields on $M$ that are tangent at each point to the corresponding leaf acts transitively on each leaf. In other words, for each leaf $L$ and each $v \in T L$ with footpoint $p$, there is $X \in \mathcal{X}_{F}$ with $X(p)=v$.

(2) $\mathcal{F}$ is transnormal; i.e., every geodesic that is perpendicular at one point to a leaf remains perpendicular to every leaf it meets.

Let $\mathcal{F}$ be a singular Riemannian foliation on a complete Riemannian manifold $M$. A leaf $L$ of $\mathcal{F}$ (and each point in $L$ ) is called regular if the dimension of $L$ is maximal; otherwise $L$ is called singular.

Typical examples of s.r.f. are the partition by orbits of an isometric action, by leaf closures of a Riemannian foliation, examples constructed by suspension of homomorphisms (see [2, 4]), and examples constructed by changes of metric and surgery (see [5]).

Received by the editors May 25, 2007.

2000 Mathematics Subject Classification. Primary 53C12; Secondary 57R30.

Key words and phrases. Singular Riemannian foliations, equifocal submanifolds, isometric actions.

The first author was supported by CNPq and partially supported by FAPESP.

(C)2008 American Mathematical Society Reverts to public domain 28 years from publication 
A particular class of s.r.f. is the one which admits sections; i.e., for each regular point $p$ the set $\Sigma:=\exp \left(\nu_{p} L_{p}\right)$ is a complete immersed submanifold that meets each leaf orthogonally.

The concept of singular Riemannian foliations with sections (s.r.f.s. for short) was introduced in 2 and continued to be studied by the authors in [1, 3, 4, 11, 12, 5], by Lytchak and Thorbergsson in 8 and recently by Gorodski and the first author in [6. In [7] Boualem dealt with a singular Riemannian foliation $\mathcal{F}$ on a complete manifold $M$ such that the distribution of normal spaces of the regular leaves is integrable. It was proved in 4 that such an $\mathcal{F}$ must be an s.r.f.s.

An s.r.f.s. includes the partitions by orbits of a polar action and the well known class of isoparametric foliations on space forms, some of them with inhomogeneous leaves.

In [10], Terng and Thorbergsson introduced the concept of equifocal submanifolds with flat sections in compact symmetric spaces in order to generalize the definition of isoparametric submanifolds in a Euclidean space.

A connected immersed submanifold $L$ of a complete Riemannian manifold $M$ is called equifocal if it satisfies the following conditions:

(1) The normal bundle $\nu(L)$ is flat.

(2) For each parallel normal field $\xi$ on a neighborhood $U \subset L$, the derivative of the map $\eta_{\xi}: U \rightarrow M$ defined by $\eta_{\xi}(x):=\exp _{x}(\xi)$ has constant rank.

(3) $L$ has sections; i.e., for each $p \in L$, the set $\Sigma:=\exp _{p}\left(\nu_{p} L_{p}\right)$, called a section, is a complete immersed totally geodesic submanifold.

There is almost an equivalence between the notions of an s.r.f.s. and equifocal submanifolds that is worked out in the authors' works [2] and [11.

On the one hand it was proved that a closed embedded equifocal submanifold induces an s.r.f.s. by taking all its parallel submanifolds ([1], [3]) if and only if there is exactly one section through every regular value of the normal exponential map of the equifocal submanifold. The global structure inherent to an s.r.f.s. was then used to generalize some results known for isoparametric submanifolds in Euclidean space.

On the other hand, it was proved in 22 that the leaves of an s.r.f.s. are equifocal (see [1] for an alternative proof). In a converse direction to the above the equifocality of an s.r.f.s. is also a very important tool in the theory of an s.r.f.s. For example, it allows us to have a Slice Theorem, singular holonomy, Weyl pseudogroups, a relation of an s.r.f.s. to transnormal maps and an extension of Weyl-invariant forms to basic forms.

While the existence of sections has interesting structural implications, it naturally restricts the number of cases that are covered. This can best be seen in the case of homogenous s.r.f. when comparing an arbitrary isometric action with a polar action. The latter is best exemplified by the action of a compact Lie group on itself by conjugation. In this paper we want to drop the condition on the existence of sections and prove that regular leaves of an s.r.f. are also equifocal. In order to make this statement precise, we will drop the first and third conditions in the definition of an equifocal submanifold, and we will also need to change the concept of parallel normal fields to foliated vector fields. Note that the restriction $\mathcal{F}_{r}$ of $\mathcal{F}$ to the regular stratum of $M$ is a regular foliation. We recall that a vector field $\xi$ in the normal bundle of the foliation over an open subset $U$ in the regular stratum is called foliated if for each vector field $Y \in \mathcal{X}_{F}$ the Lie bracket $[\xi, Y]$ also belongs 
to $\mathcal{X}_{F}$. If we consider a local submersion $\pi$ which describes the plaques of $\mathcal{F}$ in a neighborhood of a point of $L$, then a normal foliated vector field is a normal projectable/basic vector field with respect to $\pi$.

Remark 1.2. A Bott or basic connection $\nabla$ of a foliation $\mathcal{F}$ is a connection of $T M$ with $\nabla_{X} Y=[X, Y]^{\nu \mathcal{F}}$ whenever $X \in \mathcal{X}_{F}$ and $Y$ is a vector field of the normal bundle $\nu \mathcal{F}$ of the foliation. Here the superscript $\nu \mathcal{F}$ denotes projection onto $\nu \mathcal{F}$. A foliated vector field clearly is parallel with respect to the Bott connection. This connection can be restricted to the normal bundle of a leaf.

Definition 1.3. Let $L$ be a regular leaf of an s.r.f. A normal vector field along $L$ is said to be foliated if it is Bott-parallel or, in other words, if it is locally the restriction of a foliated vector field of $\mathcal{F}_{r}$ to a neighborhood $U \subset L$.

Remark 1.4. Note that if the s.r.f. admits sections, then a normal foliated vector field is a parallel normal field along each regular leaf $L$ with respect to the induced Levi-Civita connection on $\nu L$ and vice versa. In other words, in the case of sections the induced Levi-Civita connection is a Bott connection.

We are finally ready to state our result precisely.

Theorem 1.5. Let $\mathcal{F}$ be an s.r.f. on a complete Riemannian manifold $M$. Then for each regular point $p$ there exists a neigborhood $U$ of $p$ in $L_{p}$ such that

(1) For each normal foliated vector field $\xi$ along $U$ the derivative of the map $\eta_{\xi}: U \rightarrow M$, defined as $\eta_{\xi}(x):=\exp _{x}(\xi)$, has constant rank.

(2) $W:=\eta_{\xi}(U)$ is an open set of $L_{\eta_{\xi}(p)}$.

Corollary 1.6. Let $L_{p}$ be a regular leaf with trivial holonomy and let $\Xi$ denote the set of all normal foliated vector fields along $L_{p}$.

(1) Let $\xi \in \Xi$. Then $\eta_{\xi}: L_{p} \rightarrow L_{q}$ is a covering map if $q=\eta_{\xi}(p)$ is a regular point.

(2) $\mathcal{F}=\left\{\eta_{\xi}\left(L_{p}\right)\right\}_{\xi \in \Xi ;}$; i.e., we can reconstruct the singular foliation by taking all parallel submanifolds of the regular leaf $L_{p}$.

This paper is organized as follows. In Section 2 we present the propositions needed to prove the theorem. In particular we prove two propositions which contain some improvements of Molino's results on the local analysis of an s.r.f. More precisely, we review a local decomposition result and a product theorem due to Molino (see Proposition 2.2 and Proposition 2.3). In Section 3 we prove Theorem 1.5. and in Section 4 we prove Corollary 1.6.

We are grateful to Professor Gudlaugur Thorbergsson for his consistent support. We also thank the referee for helpful suggestions.

\section{Properties of AN S.R.F.}

In this section we will present the propositions needed to prove Theorem 1.5, Throughout this section we assume that $\mathcal{F}$ is an s.r.f. on a complete Riemannian manifold $M$.

We start by recalling the so-called Homothetic Transformation Lemma of Molino (see Lemma 6.2 of 9 ).

By conjugating the homothetic transformations of the normal bundle of a plaque $P$ via the normal exponential map, one defines for small strictly positive real numbers $\lambda$, a homothetic transformation $h_{\lambda}$ with proportionality constant $\lambda$ with respect to the plaque $P$. 
Proposition 2.1 ([9]). The homothetic transformation $h_{\lambda}$ sends plaque to plaque and therefore respects the singular foliation $\mathcal{F}$ in the tubular neigborhood $\operatorname{Tub}(P)$ where it is defined.

The next two propositions contain some improvements on Molino's results (compare with Theorem 6.1 and Proposition 6.5 of [9]).

Proposition 2.2. Let $g$ be the original metric on $M$ and $q \in M$. Then there exists a tubular neighborhood $\operatorname{Tub}\left(P_{q}\right)$ and a new metric $\tilde{g}$ on $\operatorname{Tub}\left(P_{q}\right)$ with the following properties:

(a) For each $x \in \operatorname{Tub}\left(P_{q}\right)$ the normal space of the leaf $L_{x}$ is tangent to the slice $S_{\tilde{q}}$ which contains $x$, where $\tilde{q} \in P_{q}$.

(b) Let $\pi: \operatorname{Tub}\left(P_{q}\right) \rightarrow P_{q}$ be the orthogonal projection. Then the restriction $\left.\pi\right|_{P_{x}}$ is a Riemannian submersion.

(c) $\mathcal{F} \cap \operatorname{Tub}\left(P_{q}\right)$ is an s.r.f.

(d) $\mathcal{F} \cap S_{\tilde{q}}$ is an s.r.f. for each $\tilde{q} \in P_{q}$.

(e) The associated transverse metric is not changed; i.e., the distance between the plaques with respect to $g$ is the same distance between the plaques with respect to $\tilde{g}$.

(f) If a curve $\gamma$ is a geodesic orthogonal to $P_{q}$ with respect to the original metric $g$, then $\gamma$ is a geodesic orthogonal to $P_{q}$ with respect to the new metric $\tilde{g}$.

Proof. Let $X_{1}, \ldots, X_{r} \in \mathcal{X}_{F}$ (i.e., vector fields that are always tangent to the leaves) so that $\left\{X_{i}(q)\right\}_{i=1, \ldots, r}$ is a linear basis of $T_{q} P_{q}$. Let $\varphi_{t_{1}}^{1}, \ldots, \varphi_{t_{r}}^{r}$ denote the associated one parameter groups and define $\varphi\left(t_{1}, \ldots, t_{r}, y\right):=\varphi_{t_{1}}^{1} \circ \cdots \circ \varphi_{t_{r}}^{r}$ where $y \in S_{q}$ and $\left(t_{1}, \ldots, t_{r}\right)$ belongs to a neighborhood $U$ of $0 \in \mathbb{R}^{r}$. Then, reducing $U$ and $\operatorname{Tub}\left(P_{q}\right)$ if necessary, one can guarantee the existence of a regular foliation $\mathcal{F}^{2}$ with plaques $P_{y}^{2}=\varphi(U, y)$. We note that the plaques $P_{z}^{2} \subset P_{z}$ and each plaque $P^{2}$ cuts each slice at exactly one point. Using the fact that $\left.\pi\right|_{P_{y}^{2}}: P_{y}^{2} \rightarrow P_{q}$ is a diffeomorphism, we can define a metric on each plaque $P_{y}^{2}$ as $\tilde{g}^{2}:=\left(\left.\pi\right|_{P_{y}^{2}}\right)^{*} g$.

Now we want to define a metric $\tilde{g}^{1}$ on each slice $S \in\left\{S_{\tilde{q}}\right\}_{\tilde{q} \in P_{q}}$. Set $D_{p}:=\nu_{p} L_{p}^{2}$ and define $\Pi: T_{p} M \rightarrow D_{p}$ as the orthogonal projection with respect to $g$. The fact that each plaque $P^{2}$ cuts each slice at one point implies that $\left.\Pi\right|_{T_{p} S}: T_{p} S \rightarrow D_{p}$ is an isomorphism. Finally we define $\tilde{g}^{1}:=\left(\left.\Pi\right|_{T_{p} S}\right)^{*} g$ and $\tilde{g}:=\tilde{g}^{1}+\tilde{g}^{2}$, meaning that $\mathcal{F}^{2}$ and the slices meet orthogonally. Items (a) and (b) follow directly from the definition of $\tilde{g}$.

To prove item (c) it suffices to prove that the plaques of $\mathcal{F}$ are locally equidistant to each other. Let $x \in S_{\tilde{q}}, P_{x}$ be a plaque of $\mathcal{F}$. We know that the plaques of $\mathcal{F}$ are contained in the leaves of the foliation by distance-cylinders $\{C\}$ with axis $P_{x}$ with respect to $g$. We will prove that each $C$ is also a distance-cylinder with axis $P_{x}$ with respect to the new metric $\tilde{g}$. These facts and the arbitrary choice of $x$ will imply that the plaques of $\mathcal{F}$ are locally equidistant to each other.

First we recall that a smooth function $f: M \rightarrow \mathbb{R}$ is called a transnormal function with respect to the metric $g$ if there exists a $C^{2}(f(M))$ function $b$ such that $g(\operatorname{grad} f, \operatorname{grad} f)=b \circ f$. Let $f: \operatorname{Tub}\left(P_{x}\right) \rightarrow \mathbb{R}$ be a smooth transnormal function with respect to the metric $g$ so that each regular level set $f^{-1}(c)$ is a cylinder $C$ with axis $P_{x}$, e.g. $f(y)=d\left(y, P_{x}\right)^{2}$. Let $\widetilde{\text { grad } f}$ denote the gradient of $f$ with respect to the metric $\tilde{g}$. It follows from the construction of $\tilde{g}$ that

$$
\widetilde{\operatorname{grad}} f=\operatorname{grad} f+l \text {, }
$$


where $l$ is a vector tangent to a plaque of $\mathcal{F}^{2}$ and in particular to a plaque of $\mathcal{F}$.

Indeed, let $v \in D_{p}$ and $w:=\left(\left.\Pi\right|_{T_{p} S}\right)^{-1}(v)$. Then

$$
\begin{aligned}
g(\operatorname{grad} f, v) & =d f(v) \\
& =d f(w) \\
& =\tilde{g}(\widetilde{\operatorname{grad}} f, w) \\
& =\tilde{g}^{1}(\widetilde{\operatorname{grad}} f, w) \\
& =g(\widetilde{\operatorname{grad}} f, \Pi w) \\
& =g(\widetilde{\operatorname{grad}} f, v) .
\end{aligned}
$$

We conclude from the arbitrary choice of $v \in D_{p}$ that $\operatorname{grad} f=\widetilde{\Pi \operatorname{grad}} f$ and hence $\widetilde{\operatorname{grad}} f=\operatorname{grad} f+l$.

Equation (2.1) implies that $f$ is a also a transnormal function with respect to the metric $\tilde{g}$, i.e.,

$$
\tilde{g}(\widetilde{\operatorname{grad}} f, \widetilde{\operatorname{grad}} f)=b \circ f \text {. }
$$

Indeed,

$$
\begin{aligned}
\tilde{g}(\widetilde{\operatorname{grad}} f, \widetilde{\operatorname{grad}} f) & =d f(\widetilde{\operatorname{grad}} f) \\
& =d f(\operatorname{grad} f) \\
& =g(\operatorname{grad} f, \operatorname{grad} f) \\
& =b \circ f .
\end{aligned}
$$

Using a local version of Q.-M. Wang's theorem [13, we conclude that each regular level set of $f$ (i.e., $C$ ) is a distance cylinder around $P_{x}$ with respect to the metric $\tilde{g}$.

Now we want to prove item (d). Set $P_{x}^{s}=P_{x} \cap S_{\tilde{q}}$ and $C^{s}:=C \cap S_{\tilde{q}}$. It suffices to note that the singular foliation $\left\{C^{s}\right\}$ is a foliation by cylinders with axis $P_{x}^{s}$ with respect to the new metric $\tilde{g}$. This follows from the fact that $\nu_{x} P_{x} \subset T_{x} S_{\tilde{q}}$ and that each geodesic orthogonal to $P_{x}$ at $x$ is contained in $S_{\tilde{q}}$ (see item (a)).

In particular we conclude that the distance between $C$ and $P_{x}$ and the distance between $C^{s}$ and $P_{x}^{s}$ with respect to the metric $\tilde{g}$ are the same.

To prove item (e) we have to prove that the distance between the cylinder $C$ and the plaque $P_{x}$ is the same for both metrics. Let $f$ be the transnormal function (with respect to $g$ ) defined above. According to Q.-M. Wang [13 for $k=f\left(P_{x}\right)$ and a regular value $c$ we have $d\left(P_{x}, f^{-1}(c)\right)=\int_{c}^{k} \frac{d s}{\sqrt{b(s)}}$. Since $f$ is also a transnormal function with respect to $\tilde{g}$ (see equation (2.2) $)$, we conclude that $d\left(P_{x}, C\right)=\tilde{d}\left(P_{x}, C\right)$, for $C=f^{-1}(c)$.

Finally we prove item (f). We consider the transnormal function $f$ above with $x=q$. In this case, equation (2.1) and the fact that grad $f \in D_{p} \cap T_{p} S$ imply that $\operatorname{grad} f=\widetilde{\operatorname{grad}} f$. On the other hand, the integral curves of the gradient of a transnormal function are geodesic segments up to reparametrization (see e.g. [13]). Therefore the radial geodesics of $P_{q}$ coincide in both metrics. This finishes the proof.

Proposition 2.3. Let $\tilde{g}$ be the metric defined in Proposition 2.2. Then there exists a new metric $g_{0}$ on $\operatorname{Tub}\left(P_{q}\right)$ so that: 
(a) We may consider the tangent space $T_{\tilde{q}} S_{\tilde{q}}$ with the metric $\tilde{g}$ and $S_{\tilde{q}}$ with the metric $g_{0}$. Then $\exp _{\tilde{q}}: T_{\tilde{q}} S_{\tilde{q}} \rightarrow S_{\tilde{q}}$ is an isometry.

(b) For this new metric $g_{0}$ we have that $\mathcal{F} \cap S_{\tilde{q}}$ and $\mathcal{F}$ restricted to $\operatorname{Tub}\left(P_{q}\right)$ are also an s.r.f.

(c) For each $x \in \operatorname{Tub}\left(P_{q}\right)$ the normal space of the leaf $L_{x}$ is tangent to the slice $S_{\tilde{q}}$ which contains $x$, where $\tilde{q} \in P_{q}$.

Remark 2.4. Clearly a curve $\gamma$ which is a geodesic orthogonal to $P_{q}$ with respect to the original metric remains a geodesic orthogonal to $P_{q}$ with respect to the new metric $g_{0}$.

Proof. Let $\Pi_{1}$ be the orthogonal projection to the slices, and recall that $\tilde{g}^{1}=\tilde{g} \circ \Pi_{1}$ and $\tilde{g}^{2}=\tilde{g} \circ d \pi$. Let $h_{\lambda}$ denote the homothetic transformation with respect to $P_{q}$. Define $g_{\lambda}=\frac{1}{\lambda^{2}} h_{\lambda}^{*} \tilde{g}^{1}+\tilde{g}^{2}$. Note that the metric $g_{\lambda}$ tends uniformly to a metric $g_{0}$ for $\lambda \rightarrow 0$. This metric $g_{0}$ restricted to $S_{\tilde{q}}$ is the induced metric on $\nu P_{\tilde{q}}$, where $\tilde{q} \in P_{q}$.

This implies that $\mathrm{L}_{\lambda}$ tends uniformly to $\mathrm{L}_{0}$, where $\mathrm{L}_{\lambda}$ is the length function. It follows then that

$$
\lim _{\lambda \rightarrow 0} d_{\lambda}(x, P)=d_{0}(x, P)
$$

where $P$ is a plaque.

Now we claim that $\mathcal{F}$ is an s.r.f. with respect to $g_{\lambda}$. Indeed, since $h_{\lambda}^{*} \tilde{g}^{2}=\tilde{g}^{2}$ and the homothetic transformation $h_{\lambda}$ sends plaque to plaque (see Proposition 2.1), it suffices to prove that $\mathcal{F}$ is an s.r.f. with respect to $\frac{1}{\lambda^{2}} \tilde{g}^{1}+\tilde{g}^{2}$. Let $f: \operatorname{Tub}\left(P_{x}\right) \rightarrow \mathbb{R}$ be a smooth transnormal function with respect to the metric $\tilde{g}$ so that each regular level set $f^{-1}(c)$ is a cylinder with axis $P_{x}$. Note that $f$ is also a transnormal function with respect to the metric $\frac{1}{\lambda^{2}} \tilde{g}^{1}+\tilde{g}^{2}$, because $\widetilde{\operatorname{grad}} f$ is tangent to the slice. Using a local version of Q.-M. Wang's theorem [13, we conclude that each regular level set of $f$ is a tube over $P_{x}$ with respect to the metric $\frac{1}{\lambda^{2}} \tilde{g}^{1}+\tilde{g}^{2}$. Therefore the plaques are equidistant to $P_{x}$, and hence we conclude that $\mathcal{F}$ is an s.r.f. with respect to $\frac{1}{\lambda^{2}} \tilde{g}^{1}+\tilde{g}^{2}$.

Finally let $x$ and $y$ be points which belong to the same plaque. Using equation (2.3) and the fact that $\mathcal{F}$ is an s.r.f. with respect to $g_{\lambda}$, we conclude that

$$
\begin{aligned}
0 & =\lim _{\lambda \rightarrow 0}\left(d_{\lambda}(x, P)-d_{\lambda}(y, P)\right) \\
& =d_{0}(x, P)-d_{0}(y, P) .
\end{aligned}
$$

The above equation implies that the plaques are locally equidistant and hence that the singular foliation $\mathcal{F}$ is Riemannian.

Now we want to prove item (c). Let $P_{x}$ be a plaque with $x \in S$. Note that for each metric $g_{\lambda}$ the normal space $H_{\lambda}$ of $P_{x}$ at $x$ (with respect to the metric $g_{\lambda}$ ) is tangent to $S$. This fact will imply that the normal space of $P_{x}$ at $x$ with respect to $g_{0}$ is also tangent to $S$. Indeed, we can find a sequence of normal spaces $H_{1 / n}$ such that $H_{1 / n}$ converge to a subspace $H_{0}$ tangent to $S$ at $x$. Then we can find a subsequence of frames $\left\{e_{i}^{n}\right\}$ which converge to a frame $\left\{e_{i}\right\}$ such that $\left\{e_{i}^{n}\right\}$ and $\left\{e_{i}\right\}$ are bases of $H_{1 / n}$ and $H_{0}$ respectively. Since

$$
\frac{1}{\lambda^{2}} h_{\lambda}^{*} \tilde{g}^{1}\left(d\left(\exp _{q}\right)_{V} Y, d\left(\exp _{q}\right)_{V} Z\right)=\tilde{g}^{1}\left(d\left(\exp _{q}\right)_{\lambda V} Y, d\left(\exp _{q}\right)_{\lambda V} Z\right),
$$

we conclude that

$$
g_{0}\left(e_{i}, l\right)=\lim _{n \rightarrow \infty} g_{1 / n}\left(e_{i}^{n}, l\right)=0
$$


where $l$ is tangent to the plaque. The last equation implies that $H_{0}$ is the normal space of $P_{x}$ at $x$ with respect to $g_{0}$.

Proposition 2.5. Let $S_{q}$ be a slice at $q$ and $\varphi: S_{q} \rightarrow S_{q}$ be the geodesic symmetry at $q$, i.e., $\varphi=\exp _{q} \circ(-\mathrm{id}) \circ \exp _{q}^{-1}$. Then the map $\varphi$ is $\mathcal{F} \cap S_{q}$ foliated; i.e., the foliation $\mathcal{F} \cap S_{q}$ is invariant by the involution $\varphi$.

Proof. It follows from Proposition 2.3 and Remark 2.4 that we can lift $\mathcal{F}$ via the exponential map in a neighborhood of $q$ to an s.r.f. of $T_{q} S_{q}$. Therefore we assume that $\mathcal{F}$ is an s.r.f. of $\mathbb{R}^{n}$ with a Euclidean metric which has $\{0\}$ as a leaf.

Lemma 2.6. The induced singular foliation on the unit sphere $\mathcal{F}^{\prime}:=\mathcal{F} \mid S^{n-1}$ is an s.r.f.

Proof. First note that every leaf of $\mathcal{F}$ that has a point in $S^{n-1}$ lies in $S^{n-1}$. Clearly $\mathcal{F}^{\prime}$ is a singular foliation. We now want to show transnormality. Let $v \in S^{n-1}$ and $\xi \in \nu_{v} L_{v} \cap T_{v} S^{n-1}$ be a unit vector. We denote by $\gamma_{\xi}$ the geodesic in $S^{n-1}$ with initial vector $\xi$. We want to show that $\xi(t):=\dot{\gamma}_{\xi}(t) \in \nu_{w} L_{w}$, where $w=\gamma_{\xi}(t)$. First we assume $t \in(0, \pi)$. Then the two unit radial vectors of $S^{n-1}$ in $v$ and $w$ span a 2-plane of $\mathbb{R}^{n}$ containing the origin. As this plane contains the straight line from $v$ to $w$, it lies in $\nu_{w} L_{w}$ by transnormality of $\mathcal{F}$. The intersection of this 2-plane with $S^{n-1}$ is exactly the geodesic $\gamma_{\xi}$. Therefore $\xi(t) \in \nu_{w} L_{w}$. This shows that $\gamma_{\xi} \mid[0, t)$ and consequently $\gamma_{\xi} \mid[0, \pi)$ is transnormal. To prove transnormality of $\gamma_{\xi} \mid[0,2 \pi)$ we repeat the argument with $w$, respectively $\xi(t)$, as our new $v$, respectively $\xi$. Since the geodesic $\gamma_{\xi}$ is closed of period $2 \pi$ only a third step is needed to show its transnormality.

Now let $v \in S^{n-1}$ and let $L_{v}$ be a leaf through $v$. Here we denote by $\nu_{v}^{\prime} L_{v}$ the normal space of $L_{v}$ in $S^{n-1}$. For any unit vector $\xi \in \nu_{v}^{\prime} L_{v}$ the geodesic $\gamma_{\xi}$ in $S^{n-1}$ meets the leaf $L_{-v}$ in the antipodal point $-v$ orthogonally, i.e., $-\xi=\dot{\gamma}_{\xi}(\pi) \in$ $\nu_{-v}^{\prime} L_{-v}$. So as vector spaces in $\mathbb{R}^{n}$ we have $\nu_{v}^{\prime} L_{v} \subset \nu_{-v}^{\prime} L_{-v}$, and by symmetry we have equality for every $v \in S^{n-1}$. In other words -id respects the normal bundle and therefore also the tangent bundle of $\mathcal{F}$. From this we conclude that -id respects $\mathcal{F}$ on $S^{n-1}$.

Corollary 2.7. Let $\gamma$ be a geodesic orthogonal to a regular leaf of an s.r.f. Then the singular points are isolated on $\gamma$.

Proof. The codimensions are invariant when restricting the foliation to the slice in the first singular point on $\gamma$. Now we apply the previous lemma.

\section{Proof of the THEOREM}

In this section we will apply the above propositions to prove the theorem. We start by proving a local version of Theorem 1.5.

Proposition 3.1. Let $\operatorname{Tub}\left(P_{q}\right)$ be a tubular neighborhood of a plaque $P_{q}, x_{0} \in$ $\operatorname{Tub}\left(P_{q}\right)$, a regular point and $\xi \in \nu P_{x_{0}}$ such that $\exp _{x_{0}}(\xi)=q$. Then we can find a neighborhood $U$ of $x_{0}$ in $P_{x_{0}}$ with the following properties:

(1) We can extend $\xi$ to a foliated normal vector field $\xi$ on $U$.

(2) The geodesic segment that is orthogonal to $P_{q}$ and contains a point $x \in U$ is $\gamma_{x}(t):=\exp _{x}((t+1) \xi)$ where $t \in[-1,1]$.

(3) $\eta_{(t+1) \xi}(U)$ is an open subset of $L_{\gamma_{x_{0}}(t)}$. 
(4) $\eta_{(t+1) \xi}: U \rightarrow \eta_{(t+1) \xi}(U)$ is a diffeomorphism for $t \neq 0$.

(5) dim rank $D \eta_{\xi}$ is constant on $U$.

Proof. The proof of item (1) is straightforward. The proof of item (2) follows from the Homothetic Transformation Lemma by Molino (Proposition 2.1).

Lemma 3.2. Let $\alpha(s)$ be a curve in $U$. Define $f(s, t)=\exp _{\alpha(s)}((t+1) \xi)$ and $J(t)=\frac{\partial f}{\partial s}(0, t)$. Then to prove items (3), (4) and (5) it suffices to prove that the Jacobi field $J$ is always tangent to the leaves.

Proof. The fact that $J$ is always tangent to the leaves, Corollary 2.7, and Proposition 2.1 imply that $\eta_{(t+1) \xi}(U) \subset L_{\gamma_{x_{0}}(t)}$, for all $t$. Proposition 2.1 implies that, for $t<0, \eta_{(t+1) \xi}(U)$ is an open subset of $L_{\gamma_{x_{0}}(t)}$ and that $\eta_{(t+1) \xi}: U \rightarrow \eta_{(t+1) \xi}(U)$ is a diffeomorphism. We conclude from the fact that $\pi: \operatorname{Tub}\left(P_{q}\right) \rightarrow P_{q}$ is a submersion that dim rank $D \eta_{\xi}$ is constant on $U$. Finally, consider $t>0$. The fact that $\pi: \operatorname{Tub}\left(P_{q}\right) \rightarrow P_{q}$ is a submersion implies that ker $D \eta_{(t+1)} \xi$ is tangent to the slice. On the other hand, $\eta_{(t+1)} \xi$ restricted to the slice is a diffeomorphism. Therefore $\left.\eta_{(t+1)} \xi\right|_{U}$ is a local diffeomorphism. It is not difficult to see that it is bijective and hence is a diffeomorphism.

In what follows we will prove that the Jacobi field $J$ defined above is always tangent to the leaves.

Let $g_{0}$ be the metric defined in Proposition 2.3. Then Remark 2.4 and item (2) imply that the Jacobi field $J$ defined in Lemma 3.2 was not changed when the metric was modified.

Now consider a geodesic segment $\gamma$ orthogonal to the leaves of $\mathcal{F}$ so that $\gamma(0)=q$ and $\gamma(1)$ is a regular point contained in $S_{q}$. It follows from Corollary 2.7 that $\gamma(t)$ is always regular for $-1 \leq t<0$ and $0<t \leq 1$.

We define $\sigma$ as the submanifold contained in $S_{q}$ which is the image by $\exp _{q}$ of a subspace and so that $\sigma$ is orthogonal to $L_{x}$ at $x$.

By Proposition 2.5, Proposition 2.3 and Proposition 2.1 we have that the plaques $P_{\gamma(t)} \cap S_{q}$ are orthogonal to $\sigma$ for $-1 \leq t \leq 1$. Then it follows from Proposition 2.3 that the plaques $P_{\gamma(t)}$ are orthogonal to $\sigma$ for $-1 \leq t \leq 1$.

Consider a geodesic segment $\beta$ so that $\beta(0)=\gamma(t)$ and $\beta$ is orthogonal to $P_{\gamma(t)}$. Then Proposition 2.3 implies that $\beta$ is contained in $S_{q}$. Since $S_{q}$ is identified with $T_{q} S_{q}$ we can consider $\beta$ as a straight line. Since $P_{\gamma(t)} \cap S_{q}$ is orthogonal to $\sigma$ and $\sigma$ is identified with a subspace, we conclude that $\beta$ is contained in $\sigma$.

Therefore $\exp _{\gamma(t)}\left(\nu(P)_{\gamma(t)} \cap B_{\epsilon}(0)\right)$ is an open set of $\sigma$. A standard argument from Riemannian geometry implies that the second form is null at $\gamma(t)$; i.e., $\sigma$ is geodesic at $\gamma(t)$. In particular the curvature tensor $R$ of $\sigma$ is the same as the ambient space at $\gamma(t)$. This fact and the fact that $R\left(\gamma^{\prime}, \cdot\right) \gamma^{\prime}$ is self-adjoint imply that $T_{\gamma(t)} \sigma$ as well $\left(T_{\gamma(t)} \sigma\right)^{\perp}$ are families of a parallel subspace along $\gamma$ which are invariant by $R\left(\gamma^{\prime}, \cdot\right) \gamma^{\prime}$.

Finally consider the $L_{x}$-Jacobi field $J$ defined in Lemma 3.2. This Jacobi field has initial conditions at $\left(T_{\gamma(1)} \sigma\right)^{\perp}$ and satisfies the Jacobi equation. So $J(t) \in\left(T_{\gamma(t)} \sigma\right)^{\perp}$ for $-1 \leq t \leq 1$.

As remarked above plaques $P_{\gamma(t)}$ are orthogonal to $\sigma$ for $-1 \leq t \leq 1$. Since $P_{\gamma(t)}$ are regular plaques for $t \neq 0$ (see Corollary 2.7), we conclude that $J(t)$ is always tangent to $P_{\gamma(t)}$.

We are finally ready to prove Theorem 1.5 
Let $L$ be a leaf of $\mathcal{F}$ and $\xi$ be a normal foliated vector field along a neighborhood $U$ of $L$. Let $p \in U$. Since singular points are isolated along $\gamma_{p}(t)=\left.\exp _{p}(t \xi)\right|_{[-\epsilon, 1+\epsilon]}$, there exists a partition $0=t_{0}<\cdots<t_{n}=1$ such that $\gamma\left(t_{i}\right)$ are the only possible singular points.

Let $P_{\gamma_{p}\left(r_{i}\right)}$ be regular plaques that belong to $\operatorname{Tub}\left(P_{\gamma_{p}\left(t_{i-1}\right)}\right) \cap \operatorname{Tub}\left(P_{\gamma_{p}\left(t_{i}\right)}\right)$, where $t_{i-1}<r_{i}<t_{i}$. Applying Proposition 3.1 we can find an open set $U_{0} \subset P_{p}$, of the plaque $P_{p}$, an open set $U_{n+1}$ of $P_{\gamma_{p}(1)}$, open sets $U_{i} \subset P_{\gamma_{p}\left(r_{i}\right)}$ of the plaques $P_{\gamma_{p}\left(r_{i}\right)}$ (for $1 \leq i \leq n$ ) and normal foliated vector fields $\xi_{i}$ along $U_{i}$ (for $0 \leq i \leq n$ ) with the following properties:

1) For each $U_{i}$, the normal foliated vector field $\xi_{i}$ is tangent to the geodesics $\gamma_{x}(t)$, where $x \in U_{0}$.

2) $\eta_{\xi_{i}}: U_{i} \rightarrow U_{i+1}$ is surjective and for $i<n$ a diffeomorphism.

3) $\left.\eta_{\xi}\right|_{U_{0}}=\left.\eta_{\xi_{n}} \circ \eta_{\xi_{n-1}} \circ \cdots \circ \eta_{\xi_{0}}\right|_{U_{0}}$.

Because dim rank $d \eta_{\xi_{i}}$ is constant on $U_{i}$, it follows that $\operatorname{dim} d \eta_{\xi}$ is constant on $U_{0}$. Since this holds for each $p \in U, \operatorname{dim} d \eta_{\xi}$ is constant on $U$. It also follows that $\eta_{\xi}(U)$ is an open set of $L_{\eta_{\xi}(U)}$.

\section{Proof of Corollary 1.6}

Let $L_{p}$ be a regular leaf with trivial holonomy and $\xi$ a normal foliated vector field along $L_{p}$. It follows from Theorem 1.5 that $\eta_{\xi}\left(L_{p}\right)$ is an open set of $L_{q}$, where $q=\eta_{\xi}(p)$. In this section we will prove that $\eta_{\xi}\left(L_{p}\right)$ is also a closed set in $L_{q}$ and hence that $\eta_{\xi}\left(L_{p}\right)=L_{q}$. In addition, when $q$ is a regular point, we will also prove that $\eta_{\xi}: L_{p} \rightarrow L_{q}$ is a covering map.

At first suppose that $L_{q}$ is a regular leaf.

For a point $z_{0} \in L_{q}$ assume that there exists a point $z_{1} \in \eta_{\xi}\left(L_{p}\right)$ which also belongs to the plaque $P_{z_{0}}$. Let $x_{\alpha}$ be a point in $L_{p}$ such that $\eta_{\xi}\left(x_{\alpha}\right)=z_{1}$. Let $\hat{\xi}_{\alpha}$ be the vector in $T_{z_{1}} M$ tangent to the geodesic $\exp _{x_{\alpha}}(t \xi)$ so that $\exp _{z_{1}}\left(\hat{\xi}_{\alpha}\right)=x_{\alpha}$. We can extend $\hat{\xi}_{\alpha}$ along the plaque $P_{z_{0}}$ to a normal foliated vector field. Theorem 1.5 implies that $\eta_{\hat{\xi}_{\alpha}}: P_{z_{0}} \rightarrow L_{p}$. Let $A$ be the set of points $z \in P_{z_{0}}$ such that $\hat{\xi}_{\alpha}(z)$ is tangent to the geodesic $\exp _{x}(t \xi)$ and $\exp _{z}\left(\hat{\xi}_{\alpha}\right)=x$ for $x \in L_{p}$. The fact that $\eta_{\xi}: L_{p} \rightarrow L_{q}$ is a local diffeomorphism (see Theorem 1.5) implies that $A$ is an open set of $P_{z_{0}}$.

Let $z_{2} \in \partial A$. By Theorem 1.5 there exist a neighborhood $U$ of $z_{2}$ in $P_{q}$ and a neighborhood $W$ of $\eta_{\hat{\xi}_{\alpha}}\left(z_{2}\right)$ in $L_{q}$ such that $\eta_{\hat{\xi}_{\alpha}}: U \rightarrow W$ is a diffeomorphism. Let $\xi_{-}:=\frac{\partial f}{\partial s}(1, z)$ be a vector field along $W$, where $f(s, z):=\exp _{z}\left(s \hat{\xi}_{\alpha}\right)$. It follows from Theorem 1.5 that $\xi_{-}$is a normal foliated vector field. Since $z_{2} \in \partial A$, we can conclude that $\xi_{-}=-\xi$ and hence $z_{2} \in A$. This implies that $A$ is a closed set of $P_{z_{0}}$. Therefore $A=P_{z_{0}}$. This means that $z_{0} \in \eta_{\xi}\left(L_{p}\right)$ and hence that $\eta_{\xi}\left(L_{p}\right)$ is a closed set in $L_{q}$.

Now we want to prove that $\eta_{\xi}: L_{p} \rightarrow L_{q}$ is a covering map for a regular point $q$. For a plaque $P_{z}$ consider all points $x_{\alpha} \in L$ so that $\eta_{\xi}\left(x_{\alpha}\right)=z$. For each $x_{\alpha}$ let $\hat{\xi}_{\alpha}$ be the vector in $T_{z} M$ tangent to the geodesic $\exp _{x_{\alpha}}(t \xi)$ so that $\exp _{z}\left(\hat{\xi}_{\alpha}\right)=x_{\alpha}$. Extend $\hat{\xi}_{\alpha}$ to a normal foliated vector field along $P_{z}$ and set $W_{\alpha}=\eta_{\hat{\xi}_{\alpha}}\left(P_{z}\right)$. As we have seen above, $\frac{\partial f}{\partial s}(1, \tilde{z})=-\xi(f(1, \tilde{z}))$ where $f(s, \tilde{z}):=\exp _{\tilde{z}}\left(s \hat{\xi}_{\alpha}\right)$ for $\tilde{z}$ in a neighborhood of $z$. This fact and the fact that $L_{p}$ has trivial holonomy imply that 
$\eta_{\xi}: W_{\alpha} \rightarrow P_{z}$ is a diffeomorphism with inverse $\eta_{\xi_{\alpha}}: P_{z} \rightarrow W_{\alpha}$. Finally, note that $\eta_{\xi}^{-1}\left(P_{z}\right)=\bigcup_{\alpha} W_{\alpha}$. We conclude that $\eta_{\xi}: L_{p} \rightarrow L_{q}$ is a covering map.

At last, suppose that $L_{q}$ is a singular leaf.

For a point $z_{0} \in L_{q}$ assume that there exists a point $z_{1} \in \eta_{\xi}\left(L_{p}\right)$ which also belongs to the plaque $P_{z_{0}}$. There exists $x_{1} \in L_{p}$ such that $z_{1}=\eta_{\xi}\left(x_{1}\right) \in P_{z_{0}}$. We can find an $s<1$ such that $y_{1}=\eta_{s \xi}\left(x_{1}\right)$ is a regular point. Since $y_{1}$ is a regular point, the plaque $P_{y_{1}}$ is an open set of $\eta_{s}\left(L_{p}\right)$. There exists a parallel normal field $\hat{\xi}$ along $P_{y_{1}}$ such that $\eta_{\hat{\xi}} \circ \eta_{s} \xi=\eta_{\xi}$. It follows that $\eta_{\hat{\xi}}\left(P_{y_{1}}\right) \subset P_{z_{0}}$. On the other hand, since the foliation is singular, the plaque $P_{y_{1}}$ intersects the slice $S_{z_{0}}$. These two facts imply that $z_{0} \in \eta_{\hat{\xi}}\left(P_{y_{1}}\right)$. Therefore $z_{0} \in \eta_{\xi}\left(L_{p}\right)$, and hence $\eta_{\xi}\left(L_{p}\right)$ is a closed set in $L_{q}$.

\section{REFERENCES}

1. M. M. Alexandrino, Integrable Riemannian submersion with singularities, Geom. Dedicata 108 (2004), 141-151. MR2112671 (2005j:53032)

2. M. M. Alexandrino, Singular Riemannian foliations with sections, Illinois J. Math. 48 (2004) No. 4, 1163-1182. MR2113670(2005i:53025)

3. M. M. Alexandrino, Generalizations of isoparametric foliations, Mat. Contemp. 28 (2005), 29-50. MR:2195188 (2006m:53041)

4. M. M. Alexandrino, Proofs of conjectures about singular Riemannian foliations, Geom. Dedicata 119 (2006) No. 1, 219-234. MR2247659 (2007g:53026)

5. M. M. Alexandrino and D. Töben, Singular Riemannian foliations on simply connected spaces, Differential Geom. and Appl. 24 (2006), 383-397. MR2231053 (2007d:53037)

6. M. M. Alexandrino and C. Gorodski, Singular Riemannian foliations with sections, transnormal maps and basic forms, Annals of Global Analysis and Geometry 32 (3) (2007), 209-223. MR.2336175

7. H. Boualem, Feuilletages riemanniens singuliers transversalement intégrables, Compos. Math. 95 (1995), 101-125. MR.1314698 (96e:53035)

8. A. Lytchak and G. Thorbergsson, Variationally complete actions on nonnegatively curved manifolds, to appear in Illinois J. Math.

9. P. Molino, Riemannian foliations, Progress in Mathematics, vol. 73, Birkhäuser, Boston, 1988. MR $932463(89 \mathrm{~b}: 53054)$

10. C.-L. Terng and G. Thorbergsson, Submanifold geometry in symmetric spaces, J. Differential Geometry 42 (1995), 665-718. MR1367405 (97k:53054)

11. D. Töben, Parallel focal structure and singular Riemannian foliations, Trans. Amer. Math. Soc. 358 (2006), 1677-1704. MR2186992(2006i:53030)

12. D. Töben, Singular Riemannian foliations on nonpositively curved manifolds, Math. Z. 255(2) (2007), 427-436. MR2262739 (2007i:53026)

13. Q.-M. Wang, Isoparametric functions on Riemannian manifolds. I, Math. Ann. 277 (1987), 639-646. MR901710 (88h:53056)

Instituto de Matemática e Estatística, Universidade de São Paulo, Rua do Matão 1010,05508 090 SÃo PAUlo, Brazil

E-mail address: marcosmalex@yahoo.de

Mathematisches Institut, Universität Zu Köln, Weyertal 86-90, 50931 Köln, Germany

E-mail address: dtoeben@math.uni-koeln.de 